\title{
Eficácia da terapia para desvios fonológicos com diferentes modelos terapêuticos $* * * * *$
}

\author{
Therapy effectiveness for phonological disorders with different \\ therapeutic approaches
}

\author{
Márcia Keske-Soares* \\ Ana Rita Brancalioni** \\ Caroline Marini ** \\ Karina Carlesso Pagliarin*** \\ Marizete Ilha Ceron****
}

*Fonoaudióloga. Doutora em Lingüística Aplicada pela Pontifícia Universidade Católica do Rio Grande do Sul. Professor Adjunto do Curso de Fonoaudiologia e do Mestrado em Distúrbios da Comunicação Humana da Universidade Federal de Santa Maria (UFSM). Endereço para

correspondência:

RST, 287 - 900 - Santa Maria - RS CEP 97060-500

(keske-soares@uol.com.br)

**Graduanda de Fonoaudiologia da UFSM. Bolsista PIBIC/CNPq.

***Fonoaudióloga. Mestranda em Distúrbios da Comunicação Humana pela UFSM. Professora Substituta do Curso de Fonoaudiologia da UFSM.

*****Fonoaudióloga. Mestranda em Distúrbios da Comunicação Humana pela UFSM. Bolsista CAPES/DS

*****Trabalho Realizado no Centro de Estudos de Linguagem e Fala (CELF) da UFSM.

Artigo Original de Pesquisa

Artigo Submetido a Avaliação por Pares

Conflito de Interesse: não

Recebido em 19.12.2007.

Revisado em 28.04.2008; 13.06.2008. Aceito para Publicação em 28.07.2008.

\begin{abstract}
Background: phonological therapy. Aim: to evaluate the effectiveness of three different therapy models regarding changes in the phonological system of subjects with different phonological disorder (PD) severity levels. Method: the research group was composed by 66 subjects, 43 males and 23 females, with ages between 4:4 and 8:2 years, who were part of a school clinic database. All subjects were evaluated, before and after a period of 15 to 25 sessions of phonological therapy, using the Child's Phonological Assessment. Based on this assessment, the severity level of the PD was determined using the Percentage Consonants Correct - PCC, and by calculating the number of Non-Acquired Segments -NAS and the percentage of Acquired Segments (AS) after treatment. The subjects were treated using the following approaches: ABAB-Withdrawal and Multiple Probes, Modified Maximum Opposition and Modified Cycles Models. For the statistical analysis the T-Test was used with a significance level of 5\%. Results: the statistical analysis indicated an increase in the PCC and in the percentage of AS, as well as a reduction in the number of NAS for all groups. These results were statistically significant for the majority of the groups. Conclusion: the results indicate that the three therapy approaches were effective in the treatment of children with PD, and were effective in treating different severity levels. Besides that, most of the changes occurred in the phonological system of the groups with more severe PD.
\end{abstract}

Key Words: Phonological Disorders; Speech; Speech Therapy; Speech Disorder.

\section{Resumo}

Tema: terapia fonológica. Objetivo: avaliar a eficácia do tratamento em três diferentes modelos de terapia quanto às mudanças no sistema fonológico de sujeitos com diferentes gravidades do Desvio Fonológico (DF). Método: a amostra constituiu-se de 66 sujeitos, com idades entre 4:4 e 8:2, sendo 43 do sexo masculino e 23 do feminino, integrantes do banco de dados de uma clínica escola. Todos foram avaliados, antes e após um período de 15 a 25 sessões de terapia fonológica, utilizando-se a Avaliação Fonológica da Criança, a partir da qual foi determinada a gravidade do DF conforme o Percentual de Consoantes Corretas - PCC, o número de Segmentos Não Adquiridos - SNA, e o percentual de Segmentos Adquiridos (SA) após o período de tratamento. Os sujeitos foram tratados pelos modelos ABAB-Retirada e Provas Múltiplas, Oposições Máximas Modificado e Ciclos Modificado. Posteriormente, realizou-se análise estatística dos dados, utilizando o Teste T para amostras iguais, considerando-se $\mathrm{p}<0,05$. Resultados: verificou-se um aumento do PCC e do percentual de SA, bem como redução do número de SNA em todos os grupos tratados pelos diferentes modelos de terapia. Estes resultados foram estatisticamente significativos para a maioria dos grupos. Conclusão: os resultados permitem afirmar que os três modelos de terapia aplicados foram eficazes no tratamento de crianças com DF, para as diferentes gravidades do desvio. Além disso, as maiores mudanças no sistema fonológico ocorreram para os grupos com DF de grau mais acentuado.

Palavras-Chave: Desvio Fonológico; Fala; Fonoterapia; Desordem de Fala. 


\section{Introdução}

A alta incidência de desvios fonológicos (DF) contribuiu para a elaboração e aplicação de diferentes modelos terapêuticos com base nas teorias lingüísticas. A aplicabilidade desses modelos e a eficácia no tratamento de alterações no desenvolvimento da fala e da linguagem vêm sendo alvo de diversos estudos ${ }^{(1-11)}$. Os modelos ABAB - Retirada e Provas Múltiplas ${ }^{(12)}$, Oposições Máximas ${ }^{(8,13)}$ e Ciclos Modificado ${ }^{(14-15)}$ foram aplicados em falantes do Português Brasileiro, os quais puderam verificar sua aplicabilidade e eficácia (3-4,6,16-21).

Williams ${ }^{(22)}$ refere que o sistema fonológico da criança, o número de sons ausentes no seu inventário fonético e fonológico e a gravidade do DF podem contribuir para a escolha do modelo terapêutico mais adequado e para a eficácia do tratamento.

A partir disso, o presente trabalho teve como objetivo comparar a eficácia do tratamento em três diferentes modelos de terapia quanto às mudanças fonológicas em diferentes gravidades do DF, no que se refere ao Percentual de Consoantes Corretas (PCC) ${ }^{(2-4)}$ e segmentos não adquiridos (SNA) inicial e final, e percentual de segmentos adquiridos (SA).

\section{Método}

Este estudo foi realizado por meio de levantamento no banco de dados do Centro de Estudos de Linguagem e Fala (CELF), de uma Instituição de Ensino Superior Federal, com projeto aprovado no Comitê de Ética em Pesquisa (CEP) institucional sob número 046/02. O Termo de Consentimento Livre e Esclarecido, assinado pelos pais e/ou responsáveis, permitia a utilização dos dados das avaliações para futuras pesquisas.

A amostra constituiu-se de 66 sujeitos, 43 do sexo masculino e 23 do feminino, com idades entre $4: 4$ e $8: 2$. Estes sujeitos foram submetidos às seguintes avaliações fonoaudiológicas antes do tratamento: linguagem compreensiva e expressiva; exame articulatório-repetitivo; sistema estomatognático; psicomotricidade; discriminação auditiva, e Avaliação Fonológica da Criança (AFC) ${ }^{(23)}$. Além disso, foram submetidos às avaliações otorrinolaringológica, audiológica e neurológica.

$\mathrm{Na}$ avaliação fonológica realizou-se coleta, transcrição e análise dos dados de fala e, posteriormente, determinação da gravidade do DF, calculado a partir do ${ }^{(24)}$

A gravidade do DF é classificada pelo PCC como:
Desvio Médio (DM), PCC de 86 a 100\%; Desvio Médio-Moderado (DMM), PCC entre 66 a 85\%; Desvio Moderado-Severo (DMS), PCC de 51 a 65\%; e Desvio Severo (DS), PCC menor que 50\%.

Além destas classificações foi verificado também o número de SNA no sistema fonológico. Considerou-se segmento ausente aquele cujo percentual de produção correta obtido estivesse em valores inferiores a $40 \%$, e segmento parcialmente adquirido, aquele com valores percentuais entre $40 \%$ e $79 \%{ }^{(25)}$, e ambos classificados como SNA. Os segmentos adquiridos são os que apresentam percentual superior a $80 \%$.

Como critério de inclusão, os sujeitos deveriam apresentar: apenas alterações no aspecto fonológico; ter recebido tratamento fonoaudiológico num período de 15 a 25 sessões, em um dos modelos estudados (ABAB-Retirada e Provas Múltiplas; Ciclos Modificado e Oposições Máximas Modificado). Os mesmos não apresentavam alterações auditivas, distorções fonéticas, queixas escolares, ou outros comprometimentos que pudessem interferir no tratamento para os desvios fonológicos.

Cada sujeito recebeu tratamento fonoaudiológico de um terapeuta-estagiário do Curso de Fonoaudiologia durante todo o processo terapêutico.

No Modelo ABAB-Retirada e Provas Múltiplas foram tratados 36 sujeitos, sendo 4 com DS, 7 com DMS, 17 com DMM, e 8 com DM. No Modelo de Oposições Máximas Modificado 17 sujeitos foram tratados, 1 com DS, 4 com DMS, 9 com DMM e 3 com DM. No Modelo de Ciclos Modificado foram tratados 13 sujeitos, sendo 3 com DMS, 9 com DMM e 1 com DM. Ressalta-se que neste modelo não houve sujeito tratado com DS.

O Modelo ABAB-Retirada e Provas Múltiplas ${ }^{(12)}$, baseia-se na hierarquia implicacional de traços distintivos, utilizando-se o Modelo Implicacional de Complexidade de Traços ${ }^{(26)}$ para a seleção dos alvos de tratamento. A terapia inicia com a coleta de dados da fala (A1), seguido de ciclos de tratamento (B1) com duração de nove sessões e, na seqüência, ocorre o Período de Retirada (A2) sem tratamento direto sobre o som-alvo, com duração de cinco sessões. Desse modo, segue-se sucessivamente o tratamento, mediante outro ciclo de tratamento (B2), seguido de outro Período de Retirada (A3). Este modelo ainda contém as Provas Múltiplas que inclui a Prova de Generalização (PG) e a Prova-Alvo Básica (PAB). A primeira tem como objetivo verificar a generalização dos traços trabalhados aos sons não treinados e é realizada 
no Período de Retirada, já a segunda serve para avaliar o progresso do som-alvo durante o ciclo de tratamento. Neste modelo todos os sujeitos foram submetidos a 2 ciclos de tratamento (18 sessões), sendo trabalhados, no máximo, dois sons.

O Modelo de Oposições Máximas Modificado (8) contrasta palavras que diferem em apenas um fonema, porém se diferenciam em dois ou mais traços distintivos. No início e no final de cada sessão, é realizado o bombardeio auditivo. Inicialmente, os pares mínimos são trabalhados pela imitação da produção do terapeuta. Quando a criança obtém $80 \%$ ou mais de produções corretas, passa-se para a fase de produção espontânea, que continua até o final do processo terapêutico considerado. Após cinco sessões de terapia, realizase uma sondagem, para verificar as generalizações. Depois de um período de 20 a 25 sessões de terapia (sem contar as de sondagem), é reavaliado o sistema fonológico da criança por meio do AFC, a fim de verificar se houve progresso no tratamento. Neste modelo os sujeitos foram submetidos de 3 a 5 ciclos de tratamento, com variações de 15 a 25 sessões, sendo trabalhados de 2 a 10 sons-alvo.

O Modelo de Ciclos Modificado ${ }^{(14)}$ é utilizado quando há muitos processos fonológicos na fala. Cada ciclo tem duração de três semanas e, em cada semana, é abordado um processo fonológico. Em cada processo, um ou dois sons-alvo são enfocados e cada um deles é trabalhado durante uma sessão de terapia, passando-se para outro som na sessão seguinte. Caso a criança tenha obtido apenas $20 \%$ de correção ou menos nas palavras-estímulo daquela sessão, repete-se o alvo. Ao final das três semanas, é realizada uma sondagem, a fim de verificar as generalizações obtidas. A criança deve atingir um percentual maior que $50 \%$ de produções corretas, passando-se, então para o segundo ciclo, que utiliza os mesmos processos fonológicos do primeiro, porém no nível de sentença. No início e no final de cada sessão, é realizado o bombardeio auditivo. Neste modelo os sujeitos foram submetidos de 2 a 3 ciclos de tratamento, com variações de 15 a 23 sessões, sendo trabalhados de 6 a 18 sons-alvo.

À exceção do Modelo ABAB-Retirada e Provas Múltiplas em que o alvo é estimulado por 9 sessões, os demais permitem que cada sessão seja repetida mais uma vez quando não há êxito na sessão anterior. Após um período de 15 a 25 sessões de terapia, os sujeitos foram reavaliados com a aplicação do instrumento AFC ${ }^{(23)}$. Foi calculado novamente o PCC ${ }^{(24)}$, determinado o número de SNA do sistema fonológico e o percentual de Segmentos Adquiridos (SA).
Foi comparado o PCC e o número de SNA inicial e final, bem como o percentual de SA após o período de terapia fonológica, nos três diferentes modelos de terapia. Os dados coletados foram tabulados e submetidos a tratamento estatístico, utilizando o Teste T para amostras iguais, considerando-se $\mathrm{p}<$ 0,05 .

\section{Resultados}

Na Tabela 1 é apresentada a descrição da comparação do PCC inicial e final, do número de SNA e, ainda, do percentual de SA após o período de tratamento estudado nos diferentes modelos de terapia, quanto à gravidade do DF. Nota-se que houve aumento do PCC final em todos os grupos com diferentes gravidades do DF tratados pelos diferentes modelos, sendo estatisticamente significante. Porém, isso não foi verificado para o DM, tratado pelo Modelo de Oposições Máximas Modificado, e para o DMS submetido ao Modelo de Ciclos Modificado.

Em relação ao número de SNA, houve redução desse valor, pois os sujeitos acrescentaram segmentos em seus sistemas fonológicos, em todos os grupos tratados nos diferentes modelos terapêuticos. Não foi verificada diferença estatisticamente significante apenas para os DS e DMS, submetidos, respectivamente, aos Modelos ABAB-Retirada e Provas Múltiplas e Ciclos Modificado.

A Tabela 2 apresenta a descrição da comparação da gravidade do DF nos diferentes modelos quanto ao PCC inicial e final. É verificada uma relação inversamente proporcional entre a gravidade e o PCC. Pode-se observar que houve, nos grupos tratados pelos Modelos ABAB-Retirada e Provas Múltiplas e Oposições Máximas, um aumento mais expressivo do PCC final no DS em relação aos demais graus.

No Modelo de Ciclos Modificado, o aumento no PCC foi maior para o DMM. A diferença entre o PCC inicial e final em todos os modelos pesquisados é menor no DM, pois, nesse grupo, observou-se menor quantidade de segmentos a serem adquiridos. O contrário se observa no DS, em que essa diferença é maior que nos demais graus, pois há um maior número de segmentos a ser adquirido no sistema fonológico.

A Tabela 3 apresenta a descrição da comparação da gravidade do DF quanto aos SNA inicial e final em cada modelo terapêutico pesquisado. Observa-se relação diretamente proporcional entre a gravidade e 
o número de SNA no sistema fonológico das crianças, pois quanto maior a gravidade do $\mathrm{DF}$, maior é a quantidade de SNA no sistema fonológico para os três modelos terapêuticos estudados. No grupo tratado pelo Modelo ABAB-Retirada e Provas Múltiplas, os sujeitos com DS apresentaram maior aquisição de segmentos que os demais grupos e no de Oposições Máximas Modificado isso foi verificado no DMM. No grupo tratado pelo Modelo de Ciclos Modificado, observou-se essa melhora nos sujeitos com DMS, sendo os resultados estatisticamente significantes.

TABELA 1. Percentual de consoantes corretas (PCC), número de segmentos não adquiridos (SNA) e percentual de segmentos adquiridos (SA) em três modelos de terapia fonológica.

\begin{tabular}{|c|c|c|c|c|c|c|c|c|c|c|c|c|}
\hline & & \multicolumn{4}{|c|}{$\begin{array}{l}\text { ABAB - Retirada e Provas Múltiplas } \\
\qquad(\mathrm{n}=36)\end{array}$} & \multicolumn{4}{|c|}{$\begin{array}{l}\text { Oposições Máximas Modificado } \\
\qquad(\mathrm{n}=17)\end{array}$} & \multicolumn{3}{|c|}{$\begin{array}{l}\text { Ciclos Modificado } \\
\qquad(\mathrm{n}=13)\end{array}$} \\
\hline & & $\begin{array}{c}\text { DS } \\
(n=4)\end{array}$ & $\begin{array}{l}\text { DMS } \\
(\mathrm{n}=7)\end{array}$ & $\begin{array}{c}\text { DMM } \\
(\mathrm{n}=17)\end{array}$ & $\begin{array}{c}\mathrm{DM} \\
(\mathrm{n}=8)\end{array}$ & $\begin{array}{c}\text { DS } \\
(n=1)\end{array}$ & $\begin{array}{l}\text { DMS } \\
(n=4)\end{array}$ & $\begin{array}{l}\text { DMM } \\
(n=9)\end{array}$ & $\begin{array}{c}\mathrm{DM} \\
(\mathrm{n}=3)\end{array}$ & $\begin{array}{l}\text { DMS } \\
(\mathrm{n}=3)\end{array}$ & $\begin{array}{l}\text { DMM } \\
(\mathrm{n}=9)\end{array}$ & $\begin{array}{c}\mathrm{DM} \\
(\mathrm{n}=1)\end{array}$ \\
\hline \multirow{3}{*}{$\operatorname{PCC}(\%)$} & PCCi & $\begin{array}{l}29,0 \\
(4,2)\end{array}$ & $\begin{array}{l}60,1 \\
(3,2)\end{array}$ & $\begin{array}{l}74,5 \\
(5,2)\end{array}$ & $\begin{array}{l}87,1 \\
(1,8)\end{array}$ & $48,4^{*}$ & $\begin{array}{l}59,6 \\
(4,0)\end{array}$ & $\begin{array}{l}74,6 \\
(6,3)\end{array}$ & $\begin{array}{l}92,4 \\
(1,8)\end{array}$ & $\begin{array}{l}62,7 \\
(4,4)\end{array}$ & $\begin{array}{l}79,5 \\
(6,6)\end{array}$ & $94,0^{*}$ \\
\hline & PCCf & $\begin{array}{c}63,9 \\
(14,4)\end{array}$ & $\begin{array}{l}75,5 \\
(8,8)\end{array}$ & $\begin{array}{l}84,6 \\
(6,5)\end{array}$ & $\begin{array}{l}96,1 \\
(1,6)\end{array}$ & $74,1 *$ & $\begin{array}{c}78,4 \\
(11,5)\end{array}$ & $\begin{array}{c}92,1 \\
(10,3)\end{array}$ & $\begin{array}{l}95,4 \\
(2,5)\end{array}$ & $\begin{array}{c}76,1 \\
(13,5)\end{array}$ & $\begin{array}{l}97,7 \\
(7,4)\end{array}$ & $97,9 *$ \\
\hline & $\mathrm{P}$ & 0,01 & 0,00 & 0,00 & 0,00 & $*$ & 0,01 & 0,00 & 0,05 & 0,09 & 0,00 & $*$ \\
\hline \multirow{3}{*}{ SNA (número) } & SNAi & $\begin{array}{l}15,5 \\
(4,2)\end{array}$ & $\begin{array}{l}10,7 \\
(1,6)\end{array}$ & $\begin{array}{c}6,8 \\
(2,2)\end{array}$ & $\begin{array}{c}2,7 \\
(1,5)\end{array}$ & $13,0 *$ & $\begin{array}{c}9,2 \\
(1,2)\end{array}$ & $\begin{array}{c}6,7 \\
(2,3)\end{array}$ & $\begin{array}{c}3,7 \\
(1,1)\end{array}$ & $\begin{array}{c}10,7 \\
(1,5)\end{array}$ & $\begin{array}{c}4,4 \\
(2,6)\end{array}$ & $3,0^{*}$ \\
\hline & SNAf & $\begin{array}{c}9,7 \\
(3,6)\end{array}$ & $\begin{array}{c}7,3 \\
(4,0)\end{array}$ & $\begin{array}{c}3,5 \\
(2,4)\end{array}$ & $\begin{array}{c}0,6 \\
(0,9)\end{array}$ & $10,0^{*}$ & $\begin{array}{c}5,7 \\
(2,9)\end{array}$ & $\begin{array}{c}2,0 \\
(2,6)\end{array}$ & $\begin{array}{c}1,7 \\
(0,6)\end{array}$ & $\begin{array}{c}5,0 \\
(3,6)\end{array}$ & $\begin{array}{c}2,4 \\
(3,2)\end{array}$ & $1,0^{*}$ \\
\hline & $\mathrm{P}$ & 0,08 & 0,04 & 0,00 & 0,00 & $*$ & 0,01 & 0,00 & 0,04 & 0,06 & 0,04 & $*$ \\
\hline \multirow[t]{2}{*}{$\mathrm{SA}(\%)$} & & $\begin{array}{c}5,7 \\
(6,4)\end{array}$ & $\begin{array}{c}3,4 \\
(4,5)\end{array}$ & $\begin{array}{c}3,2 \\
(3,1)\end{array}$ & $\begin{array}{c}2,4 \\
(1,5)\end{array}$ & $3,0^{*}$ & $\begin{array}{c}3,7 \\
(1,9)\end{array}$ & $\begin{array}{c}4,7 \\
(1,7)\end{array}$ & $\begin{array}{c}2,0 \\
(1,0)\end{array}$ & $\begin{array}{c}5,7 \\
(3,8)\end{array}$ & $\begin{array}{c}2,1 \\
(2,8)\end{array}$ & $2,0^{*}$ \\
\hline & $\mathrm{P}$ & \multicolumn{4}{|c|}{0,59} & \multicolumn{4}{|c|}{0,14} & & 0,25 & \\
\hline
\end{tabular}

TABELA 2. Gravidade do desvio fonológico em cada modelo terapêutico quanto ao Percentual de Consoantes Corretas (PCC) inicial e final.

\begin{tabular}{|c|c|c|c|c|c|c|}
\hline & \multicolumn{6}{|c|}{ Modelos Terapêuticos } \\
\hline & \multicolumn{2}{|c|}{ ABAB - Retirada e Provas Múltiplas } & \multicolumn{2}{|c|}{ Oposições Máximas } & \multicolumn{2}{|c|}{ Ciclos Modificado } \\
\hline gravidade & PCCi & PCCf & PCCi & PCCf & $\mathrm{PCCi}$ & PCCf \\
\hline severo & $29,0(4,2) \mathrm{a}$ & $63,9(14,4) \mathrm{a}$ & $48,4(*) \mathrm{a}$ & $74,1(*) \mathrm{a}$ & $* *$ & $* *$ \\
\hline moderado - severo & $60,1(3,2) b$ & $75,5(8,8) \mathrm{a}$ & $59,6(4,0) \mathrm{a}$ & $78,4(11,5) \mathrm{a}$ & $62,7(4,4) \mathrm{a}$ & $76,1(13,5) \mathrm{a}$ \\
\hline médio - moderado & $74,5(5,2) \mathrm{c}$ & $84,6(6,5) b$ & $74,6(6,3) b$ & $92,1(10,3) \mathrm{a}$ & $79,5(6,6) b$ & $97,7(7,4) \mathrm{a}$ \\
\hline médio & $87,1(1,9) \mathrm{d}$ & $96,1(1,6) \mathrm{c}$ & $92,4(1,8) \mathrm{c}$ & $95,4(2,5) \mathrm{a}$ & $94,0(*) \mathrm{b}$ & $97,9(*) \mathrm{a}$ \\
\hline $\mathrm{p}$ & 0,00 & 0,00 & 0,00 & 0,07 & 0,00 & 0,05 \\
\hline
\end{tabular}

TABELA 3. Comparação da gravidade nos três modelos terapêuticos quanto aos segmentos não adquiridos (SNA) inicial e final.

\begin{tabular}{|c|c|c|c|c|c|c|}
\hline & \multicolumn{6}{|c|}{ Modelos Terapêuticos } \\
\hline & \multicolumn{2}{|c|}{ ABAB - Retirada e Provas Múltiplas } & \multicolumn{2}{|c|}{ Oposições Máximas } & \multicolumn{2}{|c|}{ Ciclos Modificado } \\
\hline gravidade & SNAi & SNAf & SNAi & SNAf & SNAi & SNAf \\
\hline severo & $15,5(4,2) \mathrm{a}$ & $9,7(3,6) \mathrm{a}$ & $13,0(*) \mathrm{a}$ & $10,0(*) \mathrm{a}$ & $* *$ & $* *$ \\
\hline moderado - severo & $10,7(1,6) \mathrm{a}$ & $7,3(4,0) \mathrm{a}$ & $9,2(1,2) \mathrm{a}$ & $5,7(2,9) \mathrm{a}$ & $10,7(1,5) \mathrm{a}$ & $5,0(3,6) \mathrm{a}$ \\
\hline médio - moderado & $6,8(2,2) c$ & $3,5(2,4) b$ & $6,7(2,3) a, c$ & $2,0(2,6) \mathrm{a}$ & $4,4(2,6) b$ & $2,4(3,2) \mathrm{a}$ \\
\hline médio & $2,7(1,5) \mathrm{d}$ & $0,6(0,9) \mathrm{b}, \mathrm{c}$ & $3,7(1,1) b, c$ & $1,7(0,6) \mathrm{a}$ & $3,0(*) a, b$ & $1,0(*)$ \\
\hline $\mathrm{P}$ & 0,00 & 0,00 & 0,00 & 0,01 & 0,00 & 0,27 \\
\hline
\end{tabular}

Legenda das Tabelas 1, 2 e 3: PCC = percentual de consoantes corretas; PCCi = percentual de consoantes corretas inicial; PCCf = percentual de consoantes corretas final; SNA = segmentos não adquiridos; SNAi = segmentos não adquiridos iniciais; SNAf = segmentos não adquiridos finais; $\mathrm{SA}=$ segmentos adquiridos; $*=$ número não representativo de sujeitos para análise estatística; $* *=$ inexistência de dados para o grau estudado; \# = letras iguais não diferem estatisticamente; \#\# = letras diferentes diferem estatisticamente. 


\section{Discussão}

A partir da análise do PCC, do número de SNA inicial e final e do percentual de SA no sistema fonológico nas diferentes gravidades do DF, em três modelos de terapia, observou-se que estes evoluíram durante o período analisado. Tais achados concordam com os estudos de Mota et al. ${ }^{(4)}$ e Pagan e Wertzner ${ }^{(5)}$, em que a aplicação desses modelos proporcionou melhoras no sistema fonológico.

$\mathrm{Na}$ análise do PCC inicial e final, verificou-se aumento em todas as gravidades, nos diferentes modelos. Entretanto, o aumento foi maior para os menores valores de PCC, ou seja, para o DS, nos Modelos ABAB-Retirada e Provas Múltiplas e Oposições Máximas Modificado. Isso concorda com o estudo de Pagan e Wertzner ${ }^{(5)}$ que, tratando sujeitos com DF pelo Modelo de Oposições Máximas, verificaram maiores evoluções para os que apresentaram valores de PCC mais baixos.

Blanco (19) aplicou o Modelo de Ciclos Modificado em sujeitos com DMS, DMM e DM, verificou que os com DMM apresentaram maior evolução do PCC. Segundo a autora, tais achados podem ser justificados pelo fato do DMM não ser tão comprometido quanto aos com desvios mais severos, nem levemente alterado quanto o com DM, estando aqueles suscetíveis a maiores mudanças.

Essa divergência em relação à melhora do PCC para os diferentes graus pode estar relacionada aos modelos de tratamento que foram utilizados. Williams ${ }^{(22)}$ consideram que determinados modelos podem ser mais efetivos para graus específicos de DF. Além disso, os resultados obtidos podem ser justificados pelo fato da amostra de Blanco ${ }^{(19)}$ não ser composta por sujeitos com DS.

Nos grupos tratados com diferentes modelos de terapia fonológica, houve redução do número de SNA em seus sistemas fonológicos, os quais concordam com os estudos de Mota e Pereira ${ }^{(18)}$; Mota et al. (4); Blanco ${ }^{(19)}$; Barberena et al. (27) e Mota et al. (11), que relataram o aumento de segmentos no sistema fonológico após terapia utilizando modelos fonológicos, e conseqüente redução do número de SNA.

Considerando-se os Modelos ABAB-Retirada e Provas Múltiplas e Ciclos Modificado, a redução do número de SNA foi maior para os grupos com gravidade mais acentuada, por serem estes mais comprometidos, necessitando estabelecer maior número de segmentos no sistema fonológico e maiores distinções de traços ${ }^{(20)}$.

Entretanto, no grupo de sujeitos submetidos ao tratamento pelo Modelo de Oposições Máximas Modificado, a maior redução foi observada no DMM, que estabeleceu, conseqüentemente, mais segmentos ao seu sistema fonológico, corroborando com os achados de Bagetti ${ }^{(8)}$, em que as maiores mudanças fonológicas ocorreram nos DMS e DMM.

Os resultados apontam que todos os grupos tratados pelos diferentes modelos de terapia apresentaram aumento no número de SA após o período de tratamento estudado. Contudo, a análise estatística revelou não haver diferença estatisticamente significante entre os modelos de terapia aplicados. Isso mostra que todos esses modelos foram igualmente eficazes no tratamento considerando-se as variáveis estudadas. Este achado concorda com o estudo de Mota et al. ${ }^{(11)}$ no qual foram aplicados três diferentes modelos terapêuticos no tratamento do DF e todos promoveram melhoras no sistema fonológico dos grupos tratados, não havendo diferença entre eles.

Com relação à mudança do PCC, quando analisada por modelo terapêutico aplicado, embora houvesse um aumento no PCC final para todos os modelos, observou-se que a mudança foi estatisticamente significante apenas para o ABABRetirada e Provas Múltiplas. Esses achados podem ser justificados pelo fato de o grupo tratado por este modelo apresentar maior número de sujeitos com grau mais acentuado do DF.

No que se refere aos SNA, observa-se que quanto maior a gravidade do DF mais SNA os sujeitos apresentavam em seus sistemas fonológicos, para todos os modelos estudados. Corroborando o presente estudo, tem-se o trabalho de Williams ${ }^{(28)}$ que, ao examinar três grupos em relação à gravidade, observou resultado mais evidente para os sujeitos que tinham mais para aprender a partir da intervenção, ou seja, nos casos mais severos.

\section{Conclusão}

Os achados desta pesquisa permitem concluir que os três modelos de terapia aplicados foram eficazes no tratamento para as diferentes gravidades do desvio fonológico. Além disso, verificou-se que as maiores mudanças ocorridas no sistema fonológico ocorreram nos grupos que apresentavam DF de grau mais severo, que apresentavam menor PCC e maior número de segmentos não adquiridos em seu sistema fonológico. 


\section{Referências Bibliográficas}

1. Gierut JA. Treatment efficacy: functional phonological disorders in children. Am. Speech-Lang. Hear. Assoc. 1998 Fev;41:85-100.

2. Almost D, Rosenbaum P. Effectiveness of speech intervention for phonological disorders: a randomized controlled trial. Develop. Med. Child Neurology. 1998;40:319-25.

3. Keske-Soares M. Terapia fonoaudiológica fundamentada na hierarquia implicacional dos traços distintivos aplicada em crianças com desvios fonológicos [Tese]. Porto Alegre: Pontifícia Universidade Católica do Rio Grande do Sul. Doutorado em Letras; 2001.

4. Mota HB, Keske-Soares M, Ferla A, Zasso LV, Dutra LV. Estudo comparativo da generalização em três modelos de terapia para desvios fonológicos. Saúde: Revista do Centro de Ciência da Saúde. 2002 Jan-dez;28(1-2):36-47.

5. Pagan LO, Wertzner HF. Intervenção no distúrbio fonológico por meio de pares mínimos com oposição máxima. Pró-Fono R. Atual. Cient. 2002 Setdez;14(3):313-24

6. Silva KC da, Ramos APF, Wippel MLM. O Modelo de oposições máximas como instrumental terapêutico em três casos de desvios fonológicos. J Bras de Fonoaudiologia. 2002 Out-dez;3(13):292-7.

7. Garrett JLZ, Nye C. The efficacy of treatment for children with developmental speech and language delay/ disorder: a meta-analysis. J. Speech Lang. Hear. Res. 2004 Ago;47:924-43.

8. Bagetti T, Mota HB, Keske-Soares M. Modelo de oposições máximas modificado: uma proposta de tratamento para o desvio fonológico. R. Soc. Bras. Fonoaudiol. 2005 Jan-mar;10(1):36-42.

9. Crosbie S, Holm A, Dodd B. Intervention for children with severe speech disorder: a comparison of two approaches. Int. J. Lang. Comm. Dis. 2005 Oct-dec;40(4):467-91.

10. Williams AL. A systematic perspective for assessment and intervention: a case study. Adv Speech-Lang Path. 2006 Sept;8(3):245-56

11. Mota HB, Keske-Soares M, Bagetti T, Ceron MI, Melo Filha MGC. Análise comparativa da eficiência de três diferentes modelos de terapia fonológica. Pró-Fono R. Atual. Cient. 2007 Jan-abr;19(1):67-74

12. Tyler A, Figurski R. Phonetic inventory changes after treating distinctions along an implicational hierarchy. Clin. Linguist. Phon. 1994;8(2):91-107.

13. Gierut JA. The conditions and course of clinicallyinduced phonological change. J. Speech Lang. Hear. Res. 1992 Out;35:1049-63.

14. Tyler A, Edwards ML, Saxman J. Clinical application of two phonologically based treatment procedures. J. Speech Hear. Dis. 1987;(52):393-409.

15. Hodson BW. Identifying phonological patterns and projecting remediation cycles: expediting intelligibility gains of a 7 year old Australian child. Adv. Speech-Lang. Path., 2006 Sept;8(3):257-64
16. Keske M. Modelo de terapia fonológica com base na teoria dos traços distintivos para crianças com desvio fonológico evolutivo [Dissertação]. Porto Alegre: Pontifícia Universidade Católica do Rio Grande do Sul. Mestrado em Letras; 1996.

17. Pereira LF. Tratamento fonológico baseado nos contrates de oposições máximas [Dissertação]. Santa Maria: Universidade Federal de Santa Maria. Mestrado em Distúrbios da Comunicação Humana; 1999.

18. Mota HB, Pereira LF. A generalização na terapia dos desvios fonológicos: experiência com duas crianças. PróFono R. Atual. Cient. 2001 Set;13(2):141-146.

19. Blanco AP. A generalização no tratamento com o modelo de ciclos modificado em diferentes graus de severidade do desvio [Dissertação]. Santa Maria: Universidade Federal de Santa Maria. Mestrado em Distúrbios da Comunicação Humana; 2003.

20. Barberena LS. A generalização obtida pelo modelo "ABAB - Retirada e Provas Múltiplas" em diferentes graus de severidade do desvio fonológico [Dissertação]. Santa Maria: Universidade Federal de Santa Maria. Mestrado em Distúrbios da Comunicação Humana; 2005.

21. Mota HB, Bagetti T, Keske-Soares M, Pereira LF. A generalização baseada nas relações implicacionais em sujeitos submetido à terapia fonológica. Pró-Fono R. Atual. Cient. 2005 Jan-abr;17(1):99-110.

22. Williams AL. Multiple oppositions: theoretical foundations for an alternative contrastive intervention framework. Am. J. Speech-Lang. Path. 2000a Nov;9:2828.

23. Yavas M, Hernandorena CLM, Lamprecht RR. Avaliação fonológica da criança: reeducação e terapia. Porto Alegre: Artes Médicas; 1991.

24. Shriberg LD, Kwiatkowski J. Phonological disorders I: a diagnostic classification system. J. Speech Hear. Dis. 1982 Aug;47:226-41.

25. Bernhardt B. The application of nonlinear phonological theory to intervention with one phonologically disorders child. Clin. Linguist. Phon. 1992;6(1-2):123-45.

26. Mota HB. Aquisição segmental do português - um modelo implicacional de complexidade de traços [Tese]. Porto Alegre: Pontifícia Universidade Católica do Rio Grande do Sul. Doutorado em Lingüística; 1996.

27. Barberena L, Keske-Soares M, Mota HB. Generalização no tratamento com o /R/ em um caso de desvio fonológico médio-moderado. R. Soc. Bras. Fonoaudiol. 2004 Outdez;9(4):229-36.

28. Williams AL. Multiple oppositions: case studies of variables in phonological intervention. Am. J. Speech-Lang. Path. 2000b Nov;9:289-99. 